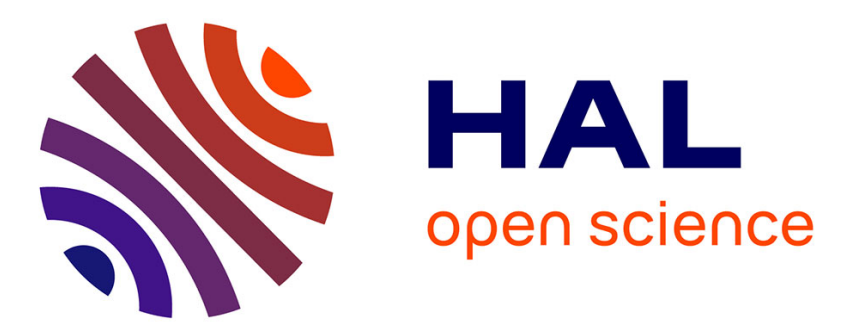

\title{
Impact of Parameters Variability on the Performances of an Implanted Antenna for Biomedical Applications
}

Shuoliang Ding, Yao Pei, Lionel Pichon, Stavros Koulouridis

\section{To cite this version:}

Shuoliang Ding, Yao Pei, Lionel Pichon, Stavros Koulouridis. Impact of Parameters Variability on the Performances of an Implanted Antenna for Biomedical Applications. 2020 14th European Conference on Antennas and Propagation (EuCAP), Mar 2020, Copenhagen (on line), Denmark. pp.1-4, 10.23919/EuCAP48036.2020.9135804 . hal-03031787

\section{HAL Id: hal-03031787 \\ https://hal.science/hal-03031787}

Submitted on 30 Nov 2020

HAL is a multi-disciplinary open access archive for the deposit and dissemination of scientific research documents, whether they are published or not. The documents may come from teaching and research institutions in France or abroad, or from public or private research centers.
L'archive ouverte pluridisciplinaire HAL, est destinée au dépôt et à la diffusion de documents scientifiques de niveau recherche, publiés ou non, émanant des établissements d'enseignement et de recherche français ou étrangers, des laboratoires publics ou privés. 


\title{
Impact of Parameters Variability on the Performances of an Implanted Antenna for Biomedical Applications
}

\author{
Shuoliang Ding ${ }^{1}$, Yao Pei $^{1}$, Lionel Pichon ${ }^{1}$, Stavros Koulouridis ${ }^{2}$ \\ ${ }^{1}$ Université Paris-Saclay, CentraleSupélec, CNRS, Laboratoire de Génie Electrique et Electronique de Paris, 91192, Gif-sur- \\ Yvette, France. \\ Sorbonne Université, CNRS, Laboratoire de Génie Electrique et Electronique de Paris, 75252, Paris, France \\ E-mail : shuoliang.ding@geeps.centralesupelec.fr \\ ${ }^{2}$ Electrical and Computer Engineering Department, University of Patras, Patras, Greece. E-mail: stavors.koulouridis@upatras.gr
}

\begin{abstract}
In this work, non-intrusive stochastic techniques are combined with 3D modeling in order to build adequate surrogate models for the evaluation of performances of a transmission link for biomedical applications. A surrogate model is appropriate to deal with uncertainties and variabilities of parameters defining the electromagnetic problem. Numerical results obtained in case of a realistic configuration involving an external patch antenna and an embedded antenna illustrate the proposed methodology.
\end{abstract}

Index Terms - implanted antennas, biomedical applications, uncertainty analysis.

\section{INTRODUCTION}

Progress in highly integrated radio-frequency (RF) circuits has a significant impact on medical implants. Miniaturized antennas not only provide wireless communication capability to transduce organ or tissue signals to a receiver outside the body and/or receive commands to adjust the implant settings but also allow continuous monitoring and therapeutic treatment without constraints in the patient's mobility. Medical implants have the advantages of being noninvasive and having high efficiency. Obtaining an optimal electromagnetic link is challenging necessitating, among others, designation of several design parameters like operational frequency, implanted device shape and maximum power deposited on the tissues while implant size needs to be minimal to avoid tissue damage and increase patient safety. For biomedical uses, several frequency bands are authorized: Medical Device Radiocommunication (MedRadio) Service band (401-406 MHz), and the Industrial, Scientific, and Medical (ISM) bands (433.1-434.8 MHz, 868-868.6 MHz, 902.8928MHz and 2.4-2.5 GHz).

Recently, many rectenna systems dedicated to biomedical application have been studied by many research teams in the world: X. Fang et al. have conducted a study at UHB (3.1$5.1 \mathrm{GHz}$ ), but losses are very high due to the high frequency [1]; the research team of M. Ali et al. has also presented their multi-resonance antenna but the antenna size is relatively big and thus hard to be implanted in-body [2].

In [3] a miniaturized circular antenna was designed to support both energy and information transmission. The antenna is embedded into a three-layer cylindrical model of arm and its performance is evaluated. It has dual resonant frequency which covers MedRadio (401- 406MHz) and ISM bands $(902-928 \mathrm{MHz})$. The wireless transmission link was analyzed and allowed to determine the amount of power that could be received from an external dipole at maximum authorized input power. However, it was observed that the performances of the implanted antenna and the characteristics of the transmission link are highly dependent on many parameters defining the problem. Geometrical parameters involved in the study like implantation depth, orientation of the antenna, distance between exciting source and embedded antenna strongly affect the overall efficiency of the system. Also physical parameters relevant to materials used for the miniaturized system or relevant to the environment (biological tissues) impact the global efficiency. For such a complex issue, a parametric analysis only relying on design of experiments and/or $3 \mathrm{~d}$ modeling tools may lead to heavy computational cost and is time consuming. In this case the introduction of stochastic tools allows to deal with the variability of all the parameters describing the electromagnetic problem [4]. Such approaches were shown to be very efficient in the framework of the determination of specific rate absorption (SAR) in biological tissues due to mobile phones at microwaves frequencies [5,6] and have already demonstrated their efficiency in case of a simplified wireless inductive power system [7].

This paper shows that a surrogate model based on polynomial chaos expansion (PCE) provides a powerful tool for optimizing the performances of the transmission link between an exciting patch antenna and an implanted antenna. In [7], Monte-Carlo method is already proved to be less efficient than the PCE method so that it will not be discussed again in this paper. The approach is applied in case of the antenna presented in [3] associated with a reflector. Such a methodology allows to identify the most significant parameters at low cost and makes easier any sensitivity analysis in view of the design stage of the system, and at the same time, save the calculation time without losing much accuracy.

\section{SCENARIO ANALYSIS}

The miniaturized antenna is designed to be implanted into human body by surgery. However, due to the huge difficulty to control the antenna's orientation, implanted 
location and depth during the surgery, an analysis of the impact of these parameters is of vital importance. The results will not only serve as an indication for surgeons to know where to pay more attention, but also anticipate the different possible cases that would occur during the transmission.

The entire power transmission scenario is shown in Fig. 1. A three-layer cylindrical human arm model (radius are: Bone 0-25mm; Muscle 25-47.5mm; Skin 47.5-50mm) is used as the simulation environment. In order to save the calculation time, the length of the arm model is set to the minimum value so that it does not affect the results. An external rectangular patch antenna radiates energy from outside the arm to the deep-implanted antenna in muscle (distance larger than $10 \mathrm{~mm}$ from skin) circular dipole. A patch antenna that resonates at $915 \mathrm{MHz}$ is used in order to be more directive than an isotropic antenna and thus reduce loss. Besides, a metallic wave reflector is placed behind the human tissue model in order to enhance the reception. The half-cylindrical reflector has length $=380 \mathrm{~mm}$, open angle $=$ $190^{\circ}$, thickness $=1.2 \mathrm{~mm}$ and is placed at $5 \mathrm{~mm}$ from the skin surface.

Three parameters rotX, rot $\mathrm{Y}$ and $\operatorname{rot} \mathrm{Z}$ represent the three possible angles (in relation with each axis respectively) of the implanted antenna according to its original position in Fig.1. The implantation depth $\mathrm{tz}$ is defined as the distance between the center of the implanted antenna and the skin surface. tx is the distance between the implanted antenna center and the symmetry plane. In the following study, these five parameters are divided into two different groups in order to reduce the computation time and complexity. Furthermore, each group consists of a series of parameters that are similar and difficult to prioritize when this wireless system is to be embedded into human body. In a practical point of view, this helps to identify the most important factor in each group when sacrifice has to be made. Therefore, the division of these five parameters into two different groups is necessary.

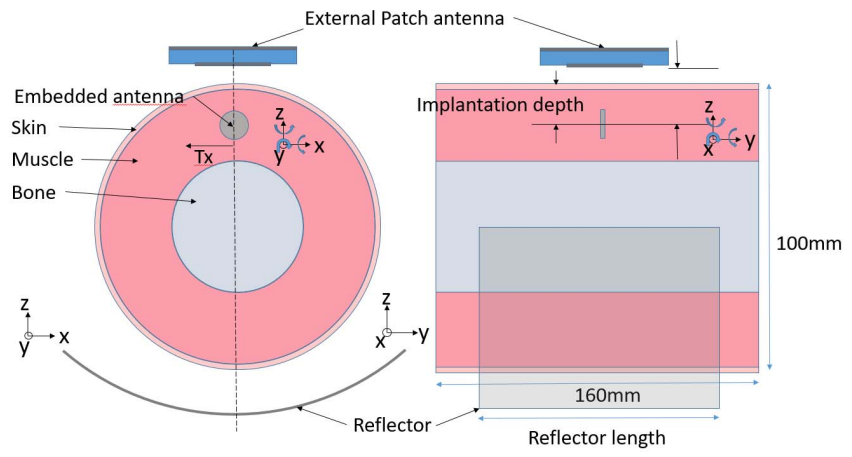

Fig. 1. Power transmission link design

All the chosen parameters have, on one hand, many accuracy uncertainties in real life, and on the other hand, have relatively strong effect on the power transmission results, and thus, meaningful to analyze.

\section{NUMERICAL RESULTS}

\section{A. Uncertainty and vairability study}

Polynomial chaos expansions (PCE) is a metamodelling technique that aims at providing an approximation of an output variable through its spectral representation on a suitable basis of polynomial functions [4]. In this chapter, a PCE model is established to simulate the varying trend of the S21 values (output) according to the different parameters mentioned in the previous chapter. The polynomial expansion is built from a design experiment (samples) deduced from 3D computations with CST Studio [8]. The following results given in this chapter are obtained by the UQLAB toolbox (Uncertainty Quantification toolbox in MATLAB) [4].

The polynomial order does not exceed the total number of samples that are taken into account. The samples in this paper are picked up uniformly. Orthogonal Matching Pursuit (OMP) method is chosen as the computing method in order to get the most optimized polynomial order.

Firstly, the trustworthiness of the method must be approved since it is used during the entire study. Therefore, the Leave-One-Out (LOO) error is calculated so as to ensure the accuracy of the results.

The LOO error is a global indicator defined as in [9]

$$
E_{L O O}=\frac{1}{N} \sum_{i=1}^{N}\left(M\left(x_{i}\right)-\mu_{\hat{Y},(-i)}\left(x_{i}\right)\right)^{2} / \operatorname{Var}[Y]
$$

where $\mu_{\hat{Y},(-i)}\left(x_{i}\right)$ is the mean predictor that was calculated with all but the $i_{\text {th }}$ sample. This value shows how much the estimated results change with the absence of one single sample point. More the LOO value is near 0 , more the method is stable and reliable. This LOO error is calculated between the estimated results and the original data.

In order to equilibrate the computation time with the results' accuracy, the polynomial order for metamodeling is decreased and set to the value with a relatively low LOO error.

Here 150 samples of different antenna orientations (angles) are calculated and 20 samples of different antenna position (distances) are analyzed separately.

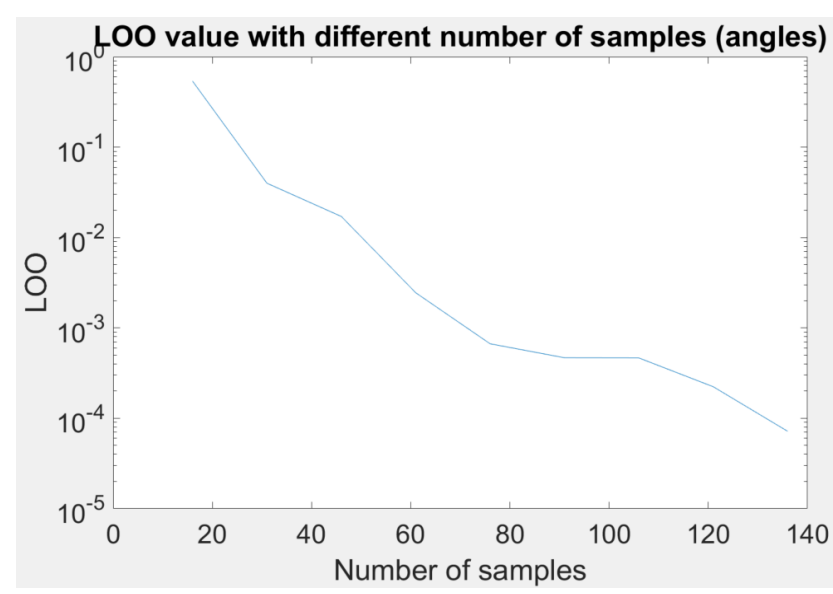

Fig. 2. LOO values with different numbers of samples calculated (angles) 


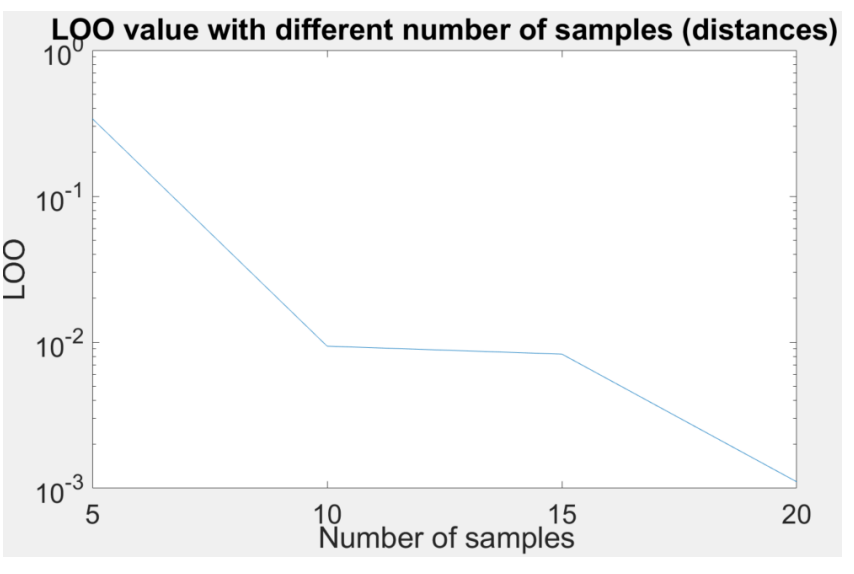

Fig. 3. LOO values with different numbers of samples calculated (distances)

Fig. 2 and Fig.3 show the relation between LOO error and number of samples taken into account. As expected, the LOO error decreases continuously as more samples are included. In the case of angles, with 50 samples ( 50 set of 3 angles), the LOO error is smaller than $1 \%$. This convergent tendency approves the trustworthiness of this surrogate model.

\section{B. Sobol Indices and sensitivity analysis}

A Sobol Index is a number that varies from 0 to 1 and who characterize the sensitivity of the output against each parameter [10]. More the index is close to 1, more the result is sensitive against this parameter. With the value of Sobol indices, it is easy to figure out which parameter to pay more attention with and which one to sacrifice. Each Sobol index of the output $\mathrm{S} 21=\mathrm{M}_{1}(\operatorname{rot} \mathrm{X}, \operatorname{rot} \mathrm{Y}, \operatorname{rotZ}), \mathrm{S} 21=\mathrm{M}_{2}(\mathrm{tx}, \mathrm{tz})$ is defined as

$$
\text { SobolIndex }=\frac{\operatorname{Var}\left[E\left[S_{21} \mid P\right]\right]}{\operatorname{Var}\left[S_{21}\right]}
$$

where $\mathrm{P}$ is one of the variables that affect the output.

The Sobol's indices are easily evaluated with a surrogate PCE model [9]. Fig. 4 and Fig. 5 shows the first order Sobol Index relevant to the different uncertainty parameters.

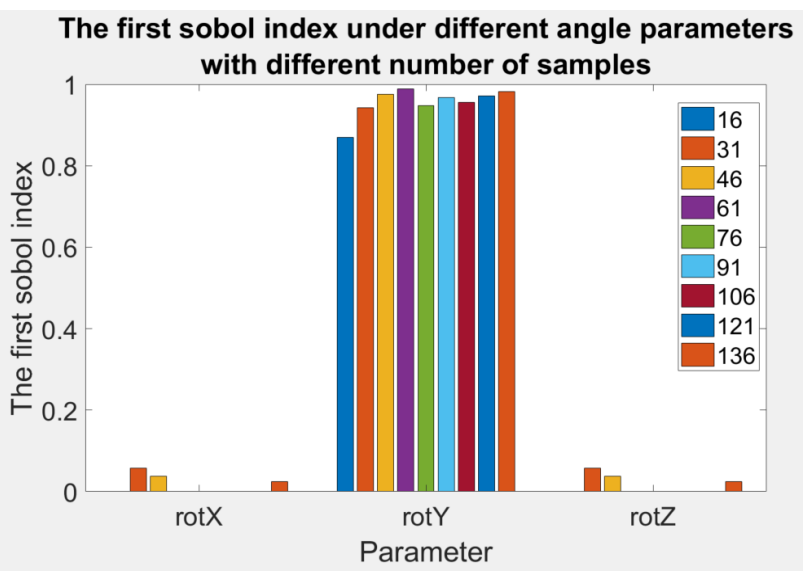

Fig. 4. Sobol indices of different angle parameters with different numbers of samples taken into account (indicated with different colors)

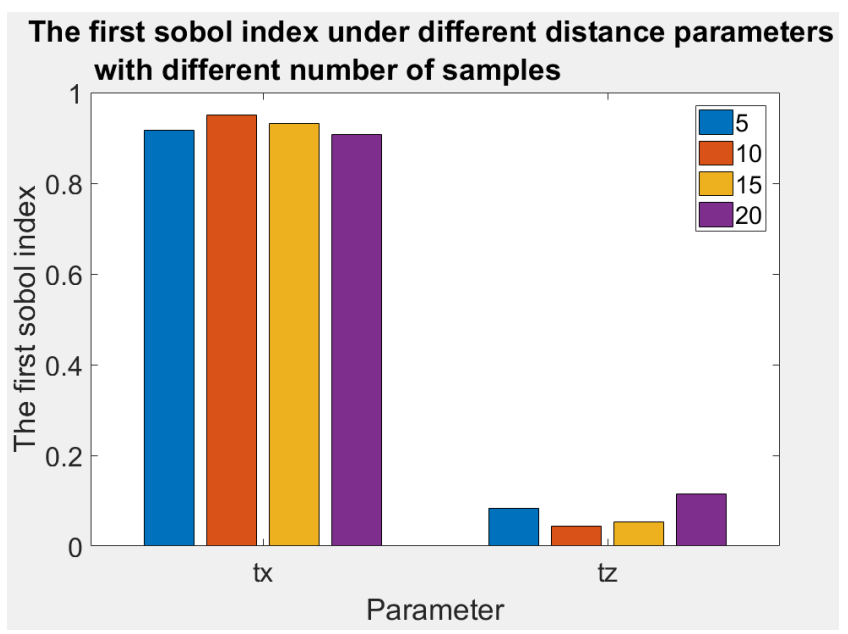

Fig. 5. Sobol indices of different parameters with different numbers of samples taken into account (indicated with different colors)

As shown in Fig. 4, the values of the first Sobol index for $\operatorname{rot} \mathrm{Z}$ and $\operatorname{rot} \mathrm{X}$ are clearly less important than that of $\operatorname{rot} \mathrm{Y}$, which shows that the rotation around $\mathrm{Y}$ axis (the antenna's symmetric axis) should be paid more attention with. Thus it is strongly suggested that the insert orientation should be marked on the antenna's surface in order to facilitate the surgery procedure. Moreover, in Fig. 5, tx has more important impact on the results than $\mathrm{tz}$, which means the surgery place should be well chosen. In contrast, the implantation depth is less important with whatever the number of samples taken into account, which corresponds to previous results in [3].

\section{Results analysis}

Previously in part A, the LOO value shows that the estimation model of the angle simulation could be established with limited number of samples. Thus these results could shorten largely the simulation time when a great number of simulation results are needed. For the 3 parameters of angle, the value predicted by the PCE estimation model with 60 samples has only less than $2 \%$ difference comparing with the reference results from simulations. For distance parameters, only 10 results are needed. Fig. 6 shows, in case of 60 angles samples (rotX, rotY, rotZ), and around the resonant frequency, the comparison between the estimated S21 value and the result obtained by the CST simulation for a random set of parameters. The same variation of S21 is shown on Fig. 7 in case of 10 distance samples $(\mathrm{tx}, \mathrm{tz})$.

As seen in Fig. 6, the predicted S21 well matched the simulation results with a maximum error of only $2 \%$. $(0.8 \%$ for distance samples). The uncertainty bars are also shown in the figures. These uncertainty bars show, for example in the case of angle calculation, the average output prediction deviation calculated by the rest 90 of the 150 data apart from the 60 sets of parameters which are already used to construct the current prediction model. 
In consequence, this surrogate model could be used to predict the real simulation results with an error tolerance smaller than $2 \%$. This could largely save the calculation time without losing much accuracy when a huge amount of results is required.

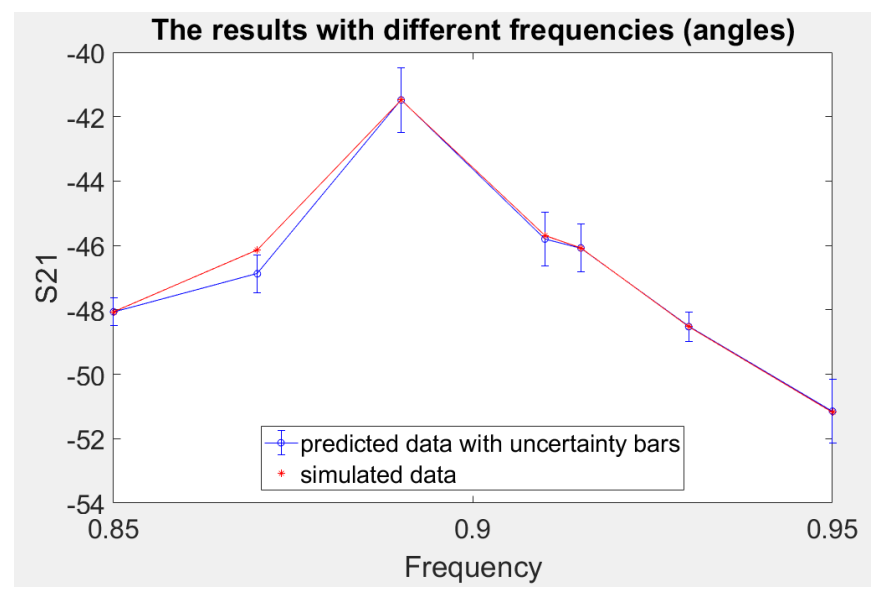

Fig. 6. Predicted results with uncertainty bar and simulated results (angle results, predicted by 60 samples)

The results with different frequencies (distances)

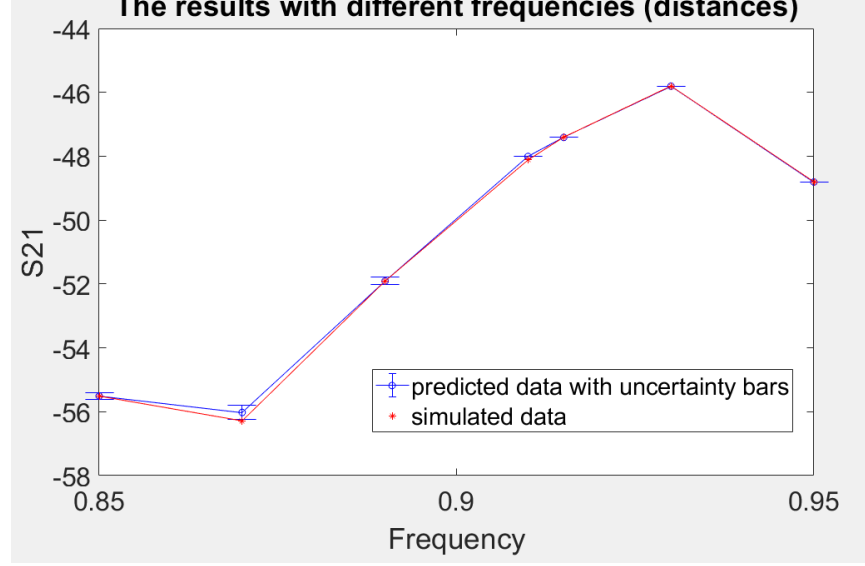

Fig. 7. Predicted results with uncertainty bar and simulated results (distance results, predicted by 10 samples)

\section{CONCLUSION}

This paper presents a methodology based on a surrogate model (PCE method) to improve the microwave transmission link calculation in a biomedical application. The studied configuration involving an exciting patch antenna and an implanted antenna is very sensitive to many geometrical parameters. A surrogate model allows to deduce at low cost the behavior of the system according to the variability of the parameters. The trustworthiness of such model is first validated and the first order Sobol indices are calculated in order to compare the impact of different parameters on the S21 results. Based on these impact factor, some advices on surgery may be given to ensure the performance. Finally, the model is used to predict the result of a random combination of parameters while deviations are controlled under $2 \%$, which proves the possibility of saving calculation time by using this method when a large number of results are required.

\section{REFERENCES}

[1] X. Fang et al., "Experimental In-Body to On-Body and In-Body to InBody Path Loss Models of Planar Elliptical Ring Implanted Antenna in the Ultra-Wide Band," 2019 13th International Symposium on Medical Information and Communication Technology (ISMICT), Oslo, Norway, 2019, pp. 1-5.

[2] Ali, M. M., Bashar, M. E. I., \& Hosain, M. K. (2017). Circural Planner Inverted-F Antenna for Implantable Biomedical Applications. 2017 2nd International Conference on Electrical \& Electronic Engineering (ICEEE), 1, 1-4.

[3] S. Ding, S.Koulouridis, L. Pichon, A Dual-Band Miniaturized Circular Antenna for Deep in Body Biomedical Wireless Applications, 13th European Conference on Antennas and Propagation (EuCAP 2019), 2019 March 31th - April 5 $5^{\text {th }}$ Krakow, Poland.

[4] S. Marelli, and B. Sudret, UQLab: A framework for uncertainty quantification in Matlab, Proc. 2nd Int. Conf. on Vulnerability, Risk Analysis and Management (ICVRAM2014), Liverpool, United Kingdom, 2014, 2554-2563.

[5] D. Voyer, F. Musy, L. Nicolas, R. Perrussel, Probabilistic methods applied to 2D electromagnetic numerical dosimetry, COMPEL, Vol. 27 No. 3, 2008, pp. 651-667.

[6] J. Silly-Carette, D. Lautru, M-F. Wong, A. Gati, J. Wiart, V. Fouad Hanna, Variability on the Propagation of a Plane Wave Using Stochastic Collocation Methods in a Bio Electromagnetic Application, IEEE Microwave and Wireless Components Letters, Volume 19, Issue 4, 2009 pp185 - 187.

[7] P. Lagouanelle, V-L Krauth, L. Pichon, Uncertainty Quantification in the Assessment of Human Exposure near Wireless Power Transfer Systems in Automotive Applications, Automotive 2019, Torino, July 2-4th, 2019Y.

[8] Computer Simulation Technology (CST) STUDIO SUITE. Ver 2017, CST AG

[9] S. Marelli and B. Sudret, UQLab user manual - Polynomial Chaos Expansions, Report UQLab-V1.1-104, Chair of Risk, Safety \& Uncertainty Qualification, ETH Zurich, 2018

[10] I. M. Sobol "Sensitivity estimates for nonlinear mathematical models," Mathematical Modelling and Computational Experiments, Vol. 1, No. 4, 407-414, 1993. 